\title{
Leiomiossarcoma de veia cava inferior: um relato de caso
}

\author{
Leiomyosarcoma of the inferior vena cava: a case report
}

Maria Silian Mandu Fonseca', Lenon Cardoso ${ }^{1}$, Gustavo Rosa de Almeida Lima ${ }^{1}$ André Poci Liberato ${ }^{2}$, Matheus Christian Silveira ${ }^{1}$, Luiz Eduardo Lima Ramos ${ }^{1}$, Eduardo Miolo Carvalho ${ }^{1}$, Júlio César Martinez ${ }^{1}$

\begin{abstract}
O leiomiossarcoma de veia cava inferior é uma neoplasia rara originária das células do músculo liso da túnica média da veia cava inferior. Neste trabalho, relata-se o caso de um paciente do sexo feminino, 54 anos, com queixa inicial de dor abdominal em flanco direito há dois anos, associada a perda de peso de $7 \mathrm{~kg}$ em dois meses, inapetência e prostração, cuja ultrassonografia de abdome total evidenciou lesão sólida, hipoecogênica e de contornos levemente bocelados na adjacência da veia cava inferior e da cabeça pancreática. Foi realizada ressecção da lesão e envio do material para análise anatomopatológica, que evidenciou leiomiossarcoma de veia cava inferior. Essas lesões têm mau prognóstico e o diagnóstico precoce e ressecção completa com margens cirúrgicas livres é a única chance de sobrevida a longo prazo.

Palavras-chave: leiomiossarcoma; veia cava inferior; neoplasias; dor abdominal.
\end{abstract}

RESUMO

\section{ABSTRACT}

The vena cava leiomyosarcoma is a rare neoplasm that is originated from the smooth muscle cells of the tunica media of the inferior vena cava. In this paper, we report the case of a 54-year-old female patient with initial complaint of abdominal pain in the right flank for two years, associated with weight loss of $7 \mathrm{~kg}$ in two months, lack of appetite and prostration. The abdominal ultrasound showed a solid and hypoechoic lesion with slightly protuberance contours in the adjacency of inferior vena cava and pancreatic head. The lesion was resected, and the material was sent for histopathological analysis, that showed leiomyosarcoma of the inferior vena cava. These lesions have a poor prognosis, and the early diagnosis and the complete resection with free surgical margins are the only chances of the long-term survival.

Keywords: leiomyosarcoma; vena cava, inferior; neoplasms; abdominal pain.

\section{INTRODUÇÃO}

Os sarcomas são tumores malignos que se originam do tecido mesenquimal. ${ }^{1,2} \mathrm{O}$ leiomiossarcoma da veia cava inferior (VCI) é uma neoplasia rara originada das células do músculo liso da túnica média, sendo relatados, até o momento, aproximadamente 300 casos no mundo desde o primeiro diagnóstico de autópsia feito por Virchow e descrito por Perl em 1871. 1,3,4

A primeira ressecção cirúrgica foi feita por Mechior em 1928. ${ }^{3,4}$ Sabe-se que esse tumor ocorre de preferência em adultos do sexo feminino numa proporção de 4:1 com idade média de 54,4 anos (variando de 15 a 83 anos). ${ }^{1,2,5}$

O quadro clínico do depende basicamente de sua localização e tamanho. ${ }^{1,2} \mathrm{O}$ crescimento do tumor é insidio- so com sintomas pouco específicos como dor abdominal, perda de peso, tumoração abdominal, febre, fraqueza, anorexia, vômitos, sudorese noturna e, eventualmente, síndrome de Budd Chiari. ${ }^{2,4,6,7}$ Atualmente, o melhor tratamento com impacto na sobrevida é a ressecção completa do tumor sem comprometimento das margens cirúrgicas. ${ }^{2,4,6,8}$ Apesar da recorrência da doença ser comum, ainda não há comprovação da eficácia dos tratamentos adjuvantes ou neoadjuvantes como quimioterapia ou radioterapia. ${ }^{7,9}$ Algumas opções de tratamento após a ressecção dos leiomiossarcomas da VCI incluem reparo primário, ligadura ou reconstrução com prótese e dependem da localização do tumor e invasão de órgãos e vasos adjacentes, principalmente das veias renais. ${ }^{10}$

'Pontifícia Universidade Católica de São Paulo, Faculdade de Ciências Médicas e da Saúde - Sorocaba (SP), Brasil. ${ }^{2}$ Hospital Municipal Doutor Mario Gatti - Campinas (SP), Brasil. Autora correspondente: Maria Silian Mandu da Fonseca - Rua Fuad Bachir Abdalla, 53, apto. 1 - Jardim Faculdade CEP: 18030-315 - Sorocaba (SP), Brasil - E-mail: silian.ma@ gmail.com

Recebido em 14/11/2016. Aceito para publicação em 02/06/2017. 


\section{RELATO DO CASO}

Paciente do sexo feminino, 54 anos, hipertensa e tabagista, com queixa inicial de dor abdominal em flanco direito há dois anos de forma intermitente, mas com agravo de intensidade há um ano. $\mathrm{O}$ quadro é associado à perda de peso $-7 \mathrm{~kg}$ em dois meses -, inapetência, prostração, epigastralgia e pirose.

Ao exame físico apresentou-se em bom estado geral, com abdome globoso, flácido, indolor e sem massas palpáveis.

Nos exames complementares, a Tomografia Computadorizada de Abdome não mostrou lesões expansivas e a Ultrassonografia Pélvica apresentou aspecto ecográfico normal. No entanto, a Ultrassonografia de Abdome Total (Figura 1) evidenciou lesão sólida, hipoecogênica e de contornos levemente bocelados na adjacência da VCI e na cabeça pancreática $4,4 \times 3 \mathrm{~cm}^{2}$.

Diante do quadro clínico e os achados dos exames de imagem, a hipótese diagnóstica inicial foi de neoplasia retroperitonial indefinida. Optou-se, então, por uma abordagem cirúrgica para a retirada da lesão (Figura 2).

No intraoperatório foi identificada uma lesão aderida na VCI com infiltração de uma camada muscular do vaso sem plano de clivagem. Foi realizada, então, secção da lesão com margem de segurança e reconstrução com prótese vascular da VCI com sucesso imediato.

A peça cirúrgica foi enviada para análise anatomopatológica que confirmou tratar-se de Leiomiossarcoma de VCI. No exame macroscópico, foi possível observar uma formação multinodular constituída de tecido firme de coloração branco acinzentada, sinalizando uma neoplasia de veia cava (neoplasia de células fusiformes de baixo grau). No estudo imunohistoquímico, os cortes histológicos apresentaram positividade para os marcadores actina de músculo liso (anticorpo 1A4) e desmina (anticorpo D33), que sãoconsistentes com leiomiossarcoma. A recuperação pós-operatória foi complicada com uma Síndrome da Resposta Inflamatória Sistêmica e insuficiência renal aguda, evoluindo com choque distributivo. No terceiro dia do pós-operatório a paciente foi a óbito.

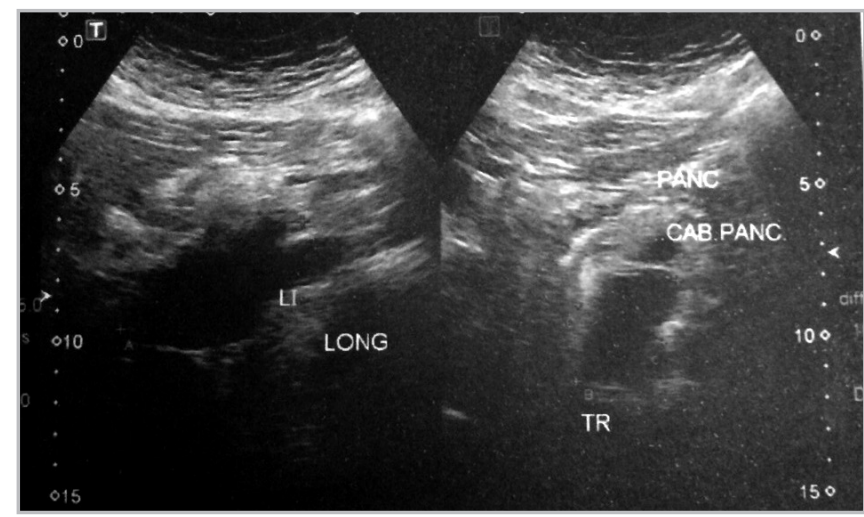

Obs.: Lesão sólida, hipoecogênica e de contornos levemente bocelados na adjacência da VCI e na cabeça pancreática $4,4 \times 3 \mathrm{~cm} 2$.

Figura 1. Ultrassonografia de abdome total.

\section{DISCUSSÃO}

O leiomiossarcoma da VCI é um tumor incomum e costuma ter mau prognóstico. Entretanto, com abordagem cirúrgica agressiva pode-se obter sobrevida longa e, eventualmente, cura, principalmente nos casos em que há ausência de metástases. ${ }^{11-13}$

O tamanho do tumor é um dos principais fatores prognósticos, sendo que a sobrevida do paciente em cinco anos varia de 30 a $53 \%$ nos pacientes submetidos a ressecção com margens livres. ${ }^{13}$ Observa-se que pode ser difícil a diferenciação entre um sarcoma de VCI e um sarcoma retroperitonial de partes moles que o envolva. ${ }^{12} \mathrm{O}$ verdadeiro sarcoma da VCI deriva das células musculares lisas do vaso e o seu padrão de crescimento pode ser intraluminal e/ou extraluminal, podendo envolver estruturas vizinhas. ${ }^{13}$ Quando o tumor tem crescimento intraluminal, pode haver dilatação da VCI e o tumor é identificado por dilatação a jusante da veia e preenchendo o lúmen. ${ }^{14}$

O lipossarcoma e o leiomiossarcoma são os sarcomas de partes moles retroperitoniais mais comuns na medicina. ${ }^{10}$ O leiomiossarcoma representa de 0,5 a $1 \%$ de todos os tumores malignos de partes moles e cerca de 5 a $10 \%$ de todos os sarcomas de partes moles, seguido pelo lipossarcoma e pelo histiocitoma fibroso maligno. O padrão típico do leiomiossarcoma na Tomografia Computadorizada de Abdome é apresentar massa lobulada, retroperitoneal, não calcificada, com ou sem áreas de baixa atenuação central por necrose ou degeneração cística, sem conteúdo de gordura e com realce heterogêneo em contraste. ${ }^{11,14}$ Segundo Webb et al. ${ }^{15}$ o sinal da VCI imperceptível no ponto de maior contato com a massa retroperitoneal tem sensibilidade de $75 \%$ e especificidade de $100 \%$ para predizer a origem da massa.

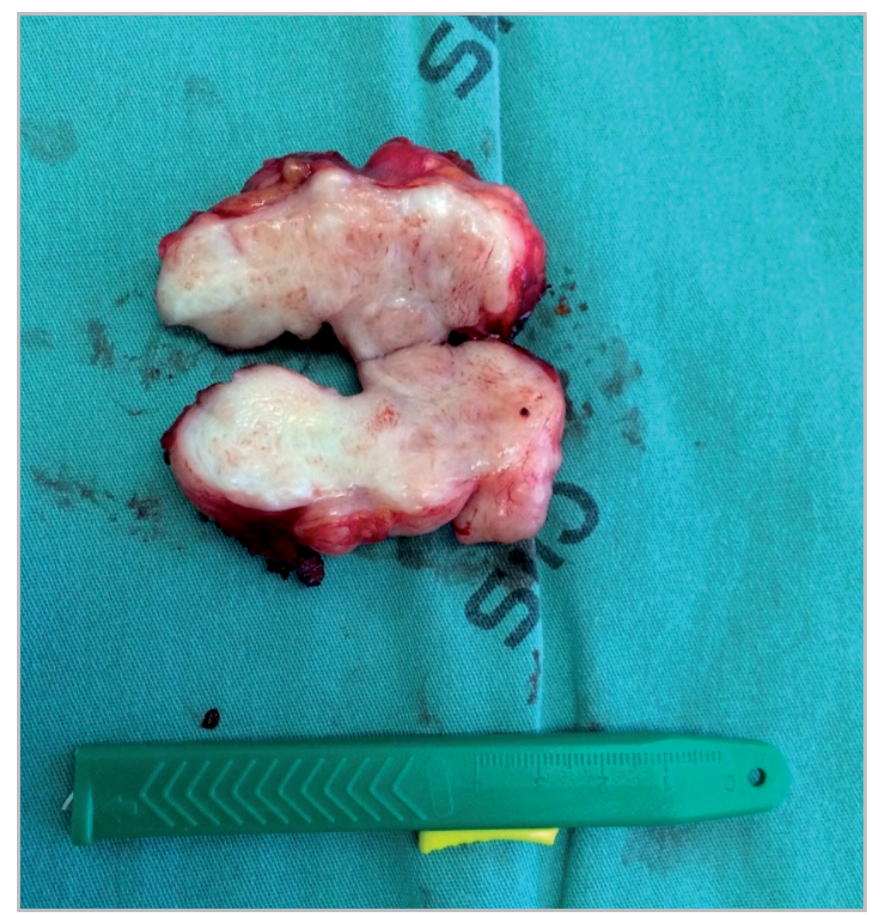

Figura 2. Peça cirúrgica: lesão sólida presente na adjacência da VCI e cabeça pancreática. 
Contudo, as estratégias para o tratamento ideal ainda permanecem incertas. ${ }^{13}$ Uma ressecção completa com margens cirúrgicas livres é a única chance de sobrevida em longo prazo. ${ }^{13,15}$ Ensaios clínicos randomizados utilizando quimioterapia e radioterapia não mostraram benefícios na sobrevida ${ }^{13}$ do paciente.

\section{CONCLUSÃO}

O caso em questão trata-se de uma condição rara, da qual não se tem conhecimento adequado para definir a conduta. Devido a essa limitação, e como pode ser frequentemente encontrado nos poucos casos como esse presentes na literatura, a conduta ativa levou a um resultado desfavorável.

\section{REFERÊNCIAS}

1. Mullen JT, DeLaney TF. Clinical features, evaluation, and treatment of retroperitoneal soft tissue sarcoma. UpToDate [Internet]. 2011 [acesso em 24 set. 2016]. Disponível em: http://www.uptodate.com/contents/ clinical-features-evaluation-and-treatment-ofretroperitoneal-soft-tissue-sarcoma?view=print

2. Mingoli A, Cavallaro A, Sapienza P, Di Marzo L, Feldhaus RJ, Cavallari N. International registry of inferior vena cava leiomyosarcoma: analysis of a world series on 218 patients. Anticancer Res. 1996;16(5B):3201-5.

3. Hollenbeck ST, Grobmyer SR, Kent KC, Brennan MF. Surgical treatment and outcomes of patients with primary inferior vena cava leiomyosarcoma. J Am Coll Surg. 2003;197(4):575-9. https://doi.org/10.1016/ S1072-7515(03)00433-2

4. Abisi A, Morris-Stiff GJ, Scott-Coombes D, Williams IM, Douglas-Jones AG, Puntis MC. Leiomyosarcoma of the inferior vena cava: clinical experience with four cases. World J Surg Oncol. 2006;4:1. https://doi. org/10.1186/1477-7819-4-1

5. Ameeri S, Butany J, Collins MJ, Nair V, Korosh K, Kandel R, et al. Leiomyosarcoma of the inferior vena cava. Cardiovasc Pathol. 2006;15(3):171-3. https://doi. org/10.1016/j.carpath.2005.08.011
6. Tan G, Chia K. An unusual case of leiomyosarcoma of the inferior vena cava in a patient with a duplicated inferior vena cava. Ann Vasc Surg. 2009;23(2):256.e138. https://doi.org/10.1016/j.avsg.2008.08.014

7. Kulaylat MN, Karakousis CP, Doerr RJ, Karamanoukian HL, O'Brien J, Peer R. Leiomyosarcoma of the Inferior vena cava: a clinicopathologic review and report of three cases. J Surg Oncol. 1997;65(3):205-17. https://doi. org/10.1002/(SICI)1096-9098(199707)65:3<205::AIDJSO11>3.0.CO;2-2

8. Bonura A, Saade C, Sharma P. Leiomyosarcoma of the inferior vena cava. Australas Radiol. 2006;50(4):395-9. https://doi.org/10.1111/j.1440-1673.2006.01611.x

9. Tameo MN, Calligaro KD, Antin L, Dougherty MJ. Primary leiomyosarcoma of the inferior vena cava: reports of infrarenal and suprahepatic caval involvement. J Vasc Surg. 2010;51(1):221-4. https:// doi.org/10.1016/j.jvs.2009.07.111

10. Brennan MF, Antonescu CR, Moraco N, Singer S. Lessons learned from the study of 10,000 patients with soft tissue sarcoma. Ann Surg. 2014;260(3):416-22. https://doi.org/10.1097/SLA.0000000000000869

11. Cantwell CP, Stack J. Abdominal aortic invasion by leiomyosarcoma. Abdom Imaging. 2006;31(1):120-2. https://doi.org/10.1007/s00261-005-0163-5

12. Drukker L, Alberton J, Reissman P. Leiomyosarcoma of the inferior vena cava: radical surgery without vascular reconstruction.VascEndovascularSurg.2012;46(8):68890. https://doi.org/10.1177/1538574412460102

13. Dew J, Hansen K, Hammon J, McCoy T, Levine EA, Shen P. Leiomyosarcoma of the inferior vena cava: surgical management and clinical results. Am Surg. 2005;71(6):497-501.

14. Narata M, Okuhata Y, Abe K, Takemoto A, Maebayashi T, Furuhashi S, et al. Primary leiomyosarcoma of the inferior vena cava: case report. Abdom Imaging. 2010;35(4):4814. https://doi.org/10.1007/s00261-009-9549-0

15. Webb EM, Wang ZJ, Westphalen AC, Nakakura EK, Coakley FV, Yeh BM. Can CT features differentiate between inferior vena cava leiomyosarcomas and primary retroperitoneal masses? Am J Roentgenol. 2013;200:205-9. https://doi.org/10.2214/AJR.11.7476

\section{Como citar este artigo:}

Fonseca MSM, Cardoso L, Lima GRA, Liberato AP, Silveira MC, Ramos LEL, et al. Leiomiossarcoma de veia cava inferior: um relato de caso. Rev Fac Ciênc Méd Sorocaba. 2018;20(2):110-2. DOI: 10.23925/1984-4840.2018v20i2a11 